\title{
Minimizing Amortized Cost of the On-demand Irrigation System in Smart Farms
}

\author{
Tiantian Xie, Zhichuan Huang, Zicheng Chi, and Ting Zhu \\ University of Maryland, Baltimore County \\ xtiant1@umbc.edu,zhihu1@umbc.edu,zicheng1@umbc.edu,zt@umbc.edu
}

\begin{abstract}
Irrigation is one of the most important food-water-energy problems in smart farms. It has a huge impact on the growth of food, while the water and energy cost for irrigation is the main cost of smart farms. In this paper, we propose an on-demand irrigation scheduling system which considers not only hourly numerical weather prediction to avoid over-irrigation but also TOU price model of electricity to minimize irrigation cost. To evaluate our design, we conduct extensive simulations with real-world trace data from Austin. The result shows that our proposed method can save $7.97 \%$ water and energy resources and reduce the amortized cost by $25.34 \%$ compared to a soil moisture based irrigation method.
\end{abstract}

\section{CCS CONCEPTS}

-Theory of computation $\rightarrow$ Scheduling algorithms; •Applied computing $\rightarrow$ Agriculture;

\section{KEYWORDS}

On-demand irrigation, Amortized cost, Smart farm

\section{ACM Reference format:}

Tiantian Xie, Zhichuan Huang, Zicheng Chi, and Ting Zhu. 2017. Minimizing Amortized Cost of the On-demand Irrigation System in Smart Farms. In Proceedings of The 3rd International Workshop on Cyber-Physical Systems for Smart Water Networks, Pittsburgh, PA USA, April 2017 (CySWATER 2017), 4 pages.

DOI: http://dx.doi.org/10.1145/3055366.3055370

\section{INTRODUCTION}

Irrigation is crucial to the growth of the plants in smart farms. It i) maintains moisture in the soil; ii) helps the growth of the roots of the crop plants; iii) is necessary for the absorption of mineral nutrients by the plants from the soil; iv) supplies two essential elements hydrogen and oxygen to the crop. Therefore, there are many related works on how to minimize the irrigation water usage, such as, soil moisture based irrigation[9], Evapotranspiration-based irrigation and measured irrigation [6]. However, they only consider the coarse grained historical or future information, such as daily moisture or evapotranspiration of the soil. In reality, daily information are still not sufficient enough to minimize water usage. For example, if the irrigation based on daily information is done just several

Permission to make digital or hard copies of all or part of this work for personal or classroom use is granted without fee provided that copies are not made or distributed for profit or commercial advantage and that copies bear this notice and the full citation on the first page. Copyrights for components of this work owned by others than ACM must be honored. Abstracting with credit is permitted. To copy otherwise, or republish, to post on servers or to redistribute to lists, requires prior specific permission and/or a fee. Request permissions from permissions@acm.org.

CySWATER 2017, Pittsburgh, PA USA

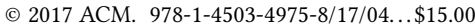

DOI: http://dx.doi.org/10.1145/3055366.3055370 hours before a rain, it is very likely that the plants would be over irrigated. Therefore, it is important to design an irrigation system that considers the hourly future information. Nowadays, numerical weather prediction (NWP) is of increasing accuracy and we can get them at nearly no cost. For example today's 5-day forecast skill is higher than $90 \%$ and 3-day' forecast near reaches $98.5 \%$ [1]. In addition, there are emerging public weather forecast API (e.g., World Weather Online and Forecast.io) providing hourly forecasts. These technologies enable us to schedule irrigation with longer lead based on hourly NWP to prevent unnecessary loss due to rainfall.

Moreover, existing irrigation systems only consider about water cost but not energy cost for irrigation. The energy cost mainly comes from pumping the water for irrigation. To reduce the energy cost, solar panels are usually deployed to collect renewable energy for irrigation. However, solar energy can only be harvested during certain period of time, we may still need energy from traditional power grid[10]. Therefore, it is important to minimize the electricity bills for irrigation. Time-of-use (TOU) electricity pricing model is applied in most of the states in United States, which means that the electricity price is different at different hours during a day. TOU pricing model has been studied in several researches to minimize cost[3]. If the irrigation system could cache energy at low-peak time instead of high-peak time when solar energy is not enough to do the irrigation, the energy cost can be reduced. Moreover, if the design is well planned, the average amortized cost of solar energy could be even lower than off-peak grid price, especially if we can replace the energy caching battery with one that contributes less to the amortized cost of solar energy (e.g., caching potential energy in water tank). Then, the schedule of caching low cost energy for irrigation could help farm owners to make their investment back earlier. However, most of current systems do not consider TOU models, which gives up the chance to reduce their electricity bills.

In this paper, we propose an on-demand irrigation scheduling system which not only utilizes NWP but also the TOU model for irrigation. The on-demand irrigation system controls when to irrigate based on the optimization over real-time energy demand estimation and solar energy prediction and when to cache energy and water. The key ideas of on-demand irrigation system are (i) the ability to identify the irrigation energy demand time and amount while avoiding possible water and energy loss due to future rainfalls, and (ii) the ability to conservatively estimate solar energy output that shifts less energy utilization to TOU high-peak pricing time. Because nearly all scheduling inputs are estimated or predicted, it is very challenging to construct an optimal on-demand irrigation scheduling method. To minimize the impact of prediction errors, we apply an adaptive prediction mechanism to update the prediction parameters. Specifically, our major contributions are: 


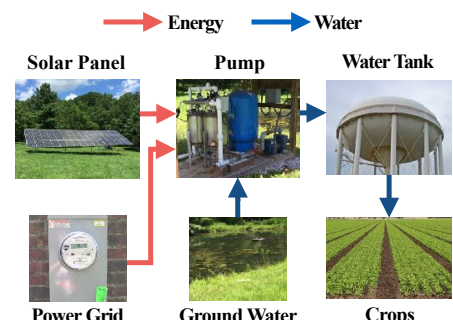

(a) System architecture.

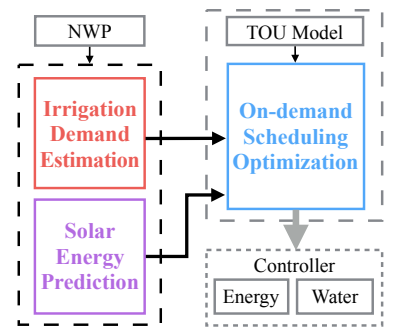

(b) On-demand irrigation system.
Figure 1: On-demand scheduling system overview.

1. To the best of our knowledge, this is the first irrigation system that provides an on-demand scheduling based on energy demand estimation and solar energy output prediction in a long lead time using hourly NWP and TOU to minimize the amortized cost.

2. Since most of the scheduling inputs are based on prediction, we design an adaptive prediction mechanism to update prediction parameters based on the historical prediction errors. A novel penalty mechanism that counters over-prediction is designed to minimize the impact.

3. We evaluated our proposed system extensively with realworld trace data. The result shows that our proposed method not only minimizes the amortized cost for irrigation by $25.34 \%$ when compared to soil moisture based irrigation but also saves the actual water and energy resources by $7.97 \%$.

\section{DESIGN}

Fig. 1a illustrates a typical crop farm's physical architecture. A pump pumps ground water using energy from solar panel or power grid. Then, the water is used to irrigate the field or stored in a water tank for later use. In this paper, we design an on-demand irrigation system with three major components (shown in Fig. 1b) to control the energy and water flow to meet the irrigation demand with minimal amortized cost.

In each scheduling period, Irrigation Demand Estimation estimates the time and energy required for the next irrigation based NWP and the soil moisture information. Solar Energy Prediction predicts solar energy distribution for the scheduling period. Then, On-demand scheduling Optimization utilizes the TOU model and these information to search for the optimal control signals. The three components are described below. Notations used in this paper are detailed in Table 1.

\subsection{Irrigation Demand Estimation}

Irrigation demand estimation is to estimate the time and amount of energy for the next irrigation.

In this paper, we denote $\lambda$ as the water scheduling policy for the crops growing in the field, which determines the starting irrigation moisture level, $\mathrm{m}^{s}$, the target moisture level, $\mathrm{m}^{t}$ and the soil moisture tolerance $\zeta$ for irrigation. $\zeta$ is to make full use of hourly NWP to avoid the loss of irrigation just hours before precipitation.

Definition 1. Energy demand time is when the soil moisture level is first below the irrigation starting level in the estimated soil moisture sequence while there is no moisture increase within the moisture tolerance after it. Energy demand amount is the very

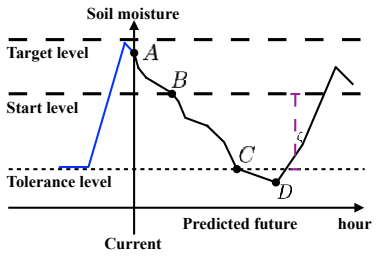

(a) Energy demand time is at B.

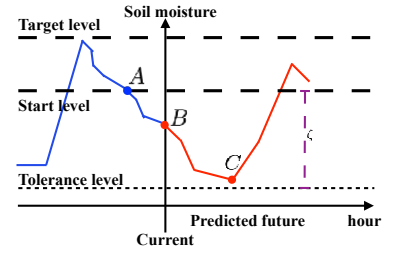

(b) No energy demand.
Figure 2: Two examples of energy demand estimation. amount of energy in need to raise the soil moisture level to the target moisture level.

For example, Fig. 2 shows two scenarios of determining the energy demand time. In figure $2 \mathrm{a}$, the energy demand time is at $\mathrm{B}$ since $m_{t_{B}}$ is the first moisture value below the irrigation start level $\left(\mathrm{m}^{s}\right)$ and there is no moisture level rising between B and C, until D that is below the tolerance level. The demand energy is the amount of energy to irrigate the field to raise the soil moisture level at B to the target soil moisture level $\left(m^{t}\right)$. In figure $2 \mathrm{~b}$, although $m_{t_{0}}$ at B is below $m^{s}$, there is no energy demand because the estimated soil moisture rises at $\mathrm{C}$ before crossing the tolerance level.

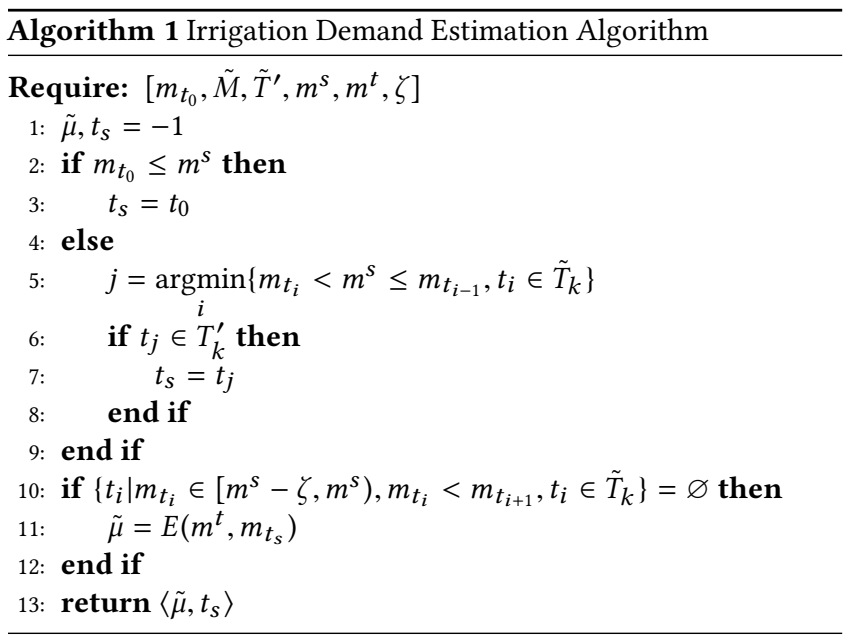

In general, we get the estimated energy demand tuple $\left\langle\tilde{\mu}, t_{s}\right\rangle$ by applying $\lambda$ to the current moisture level $\left(m_{t_{0}}\right)$ and the moisture sequence $(\tilde{M})$ estimated from future precipitation as $\left\langle\tilde{\mu}, t_{s}\right\rangle=\lambda\left(m_{t_{0}}, \tilde{M}\right)$. The detailed algorithm is shown in Algorithm 1. We first initialize $\tilde{\mu}, t_{s}$ as -1 (Line 1). If the current soil moisture level is lower than the start moisture level, then we have $t_{s}=t_{0}$ (Lines 2-3). Otherwise, we find the first time that the soil moisture level will be lower than the start moisture level (Lines 4-5). If $t_{j} \in T_{k}^{\prime}$, then we have $t_{s}=t_{j}$ (Lines 6-9). In the next step, if there is no soil moisture raise, we estimate the energy demand by $\tilde{\mu}=E\left(m^{t}, m_{t_{s}}\right)$ (Lines 10-12), where $E(\cdot)$ is a function to estimate the energy requirement to raise the soil moisture from $t_{s}$ to the target level. Finally, we return the estimated irrigation demand $\left\langle\tilde{\mu}, t_{s}\right\rangle$ (Line 13).

\subsection{Solar Energy Prediction}

There are several studies in the literature for solar energy prediction[4, $7,8]$. To predict the solar energy, we use a similar solar energy prediction method utilized in [8] - a support vector regression (SVR) 


\begin{tabular}{|c|l|c|l|}
\hline Notations & Definitions & Notations & Definitions \\
\hline$\tilde{T}_{k}$ & The total number of intervals (hour) & $\tilde{M}$ & The estimated soil moisture sequence \\
$s(t), \hat{s}(t)$ & The predicted and actual energy output for solar panel & $s(t), u(t)$ & The energy from the panel/grid \\
$\sigma_{t_{0}}$ & The energy currently stored in water tank & $p_{u}(t), p_{s}(t)$ & The cost of energy from panel/grid \\
$\tilde{\mu}$ & The energy demand at $t_{s}$ & $C$ & The virtual energy capacity in water tank \\
$\alpha$ & The maximum energy capacity of the irrigation system & $s_{\max }$ & The maximum energy output from the panel \\
$\beta$ & The under scale parameter & $\lambda$ & The irrigation policy \\
\hline
\end{tabular}

\section{Table 1: Definitions of notations.}

based method. However, all weather information are first multiplied by the cloudless irradiance as how the features are processed in [4] to allow the same weather parameter to influence the energy prediction in the same portion.

Since we are predicting in long lead times (e.g., 3 or 5 days), overpredicting the solar energy increases more chances of utilizing power grid with high electrical price, thus increasing the total amortized cost. In order to counter this over-prediction problem, we adaptively update an under scale penalty parameter based on the output of the SVR over the recent history data to counter the overpredicting problem. To achieve this, we define the error function based on mean absolute percentage error (MAPE) that needs to be optimized during each update as Eq.1:

$$
\text { error }=\frac{100}{n} \cdot \sum_{t=1}^{n} U\left(\frac{\beta \cdot s_{t}-\hat{s_{t}}}{s^{\prime}}\right)
$$

where $s_{t}$ is the predicted value and $\hat{s_{t}}$ is the observed value at time $t . s^{\prime}$ is the average value of $\hat{s_{t}}$ over time $t$ instead of $\hat{s_{t}}$. Because we may run into situation of dividing by zero (e.g., Solar energy output from the solar panel is $0 \mathrm{kWh}$ at night). $\beta$ is the under scale penalty parameter and the penalty function $U(\cdot)$ is defined as

$$
U\left(d_{s}\right)= \begin{cases}\left|d_{s}\right|, & d s \leq 0 \\ d_{s}{ }^{2}+d_{s}, & d s>0\end{cases}
$$

where $d_{s}$ is the difference of the predicted and the observed value. This function enables the optimizer to find the best parameter $\beta$ that counters the over-predicting problem.

\subsection{On-demand Scheduling Optimization}

The rationale behind the on-demand energy scheduling optimization is to minimize the amortized cost of grid and solar electricity for the next irrigation based on (i) the estimated energy demand time and amount, (ii) estimated solar energy, (iii) TOU pricing model and (iv) potential energy in the virtual energy storage (water tank). The problem is formulated as:

$$
\begin{array}{lr}
\min _{u, s} & \cos t(u, s)=\sum_{t \in \tilde{T}^{\prime}}\left(p_{u}(t) \cdot u(t)+p_{s}(t) \cdot s(t)\right) \\
\text { s.t. } & u(t) \cdot s(t)=0, t \in \tilde{T}^{\prime} \\
& \sum_{t \in \tilde{T}^{\prime}}(u(t)+s(t))+\sigma_{t_{0}} \in[\tilde{\mu}, C] \\
\text { var. } & u(t) \in[0, \alpha], t \in \tilde{T}^{\prime} \\
& s(t) \in\left[0, \min \left(s_{\text {max }}, \alpha\right)\right], t \in \tilde{T}^{\prime}
\end{array}
$$

Here $\tilde{T}^{\prime}=\left\{t_{1}, \ldots, t_{s}\right\}$ is the time period for optimization, which is determined by the energy demand time. $u(t)$ is the electricity used from the public grid at the cost of $p_{u}(t) . s(t)$ is the electricity used from the solar energy at the cost of $p_{s}(t)$. $C$ is the virtual energy capacity in the virtual energy storage. $\alpha$ is the maximum amount of energy that could be cached into the virtual energy storage during each one-hour interval.

Constraint (4) ensures that there will only be one energy source at a time either from the public grid or the solar panel. Constraint (5) guarantees that the energy demand amount $\tilde{\mu}$ could be met before energy demand time comes at $t_{s}$. This problem can be reformulated to a problem that can be solved using integer programming. We use CPLEX to solve this problem. In practice, hourly weather prediction data is provided no more than 200 hours from any weather forecast center, thus the dimension of $\tilde{T}^{\prime}$ is small. Therefore, the time complexity of the optimization algorithm is low.

After the scheduling optimization, the energy source of the pumper in the following time slot, $t_{1}$, is determined as:

$$
\text { switch }= \begin{cases}\text { grid, } & \text { when } u\left(t_{1}\right) \neq 0 \\ \text { panel, } & \text { when } s\left(t_{1}\right) \neq 0 \\ \text { off, } & \text { when } u\left(t_{1}\right)+s\left(t_{1}\right)=0\end{cases}
$$

And when the demanding time is the current time, the field starts to irrigate until the soil moisture of the field reaches the target soil moisture level.

\section{EVALUATION}

In this section, we evaluate the performance of our proposed ondemand irrigation system.

\subsection{Setup}

For the input from NWP to the solar energy prediction, we used one year 1) soil moisture information and cloudless irradiance (NOAA), 2) numerical weather information (World Weather Online) including cloud cover, dew point, humidity, precipitation ( $\mathrm{mm}$ ), pressure, temperature, visiblility, windspeed gust/miles; 3) actual solar energy output (Pecan Street) near Austin between 2014 and 2015. In each one-hour scheduling slot to predict solar energy with a lead of 120 hours, the previous two months' data are used to train an SVR model to predict the solar energy output. The nearest six day information is used to estimate the under scale parameter. In the trace driven simulation, we use the algorithm proposed in 2.1 to estimate the energy demand and time. The energy demand is determined by an energy water ratio of $0.5 \mathrm{kwh} / \mathrm{m}^{3}$ while the water demand is calculated based on the current moisture and the target moisture.

In the on-demand scheduling optimization, we use a three-rateperiod TOU price model [5] with off-peak $(0.1 \$ / \mathrm{kwh})$, partial-peak $(0.15 \$ / \mathrm{kwh})$ and peak $(0.20 \$ / \mathrm{kwh})$ for the power grid. Since the solar panel is used for a long time, the device cost can be averaged as the operational cost of solar panel. We set the solar energy cost lower than the off-peak cost as $0.08 \$ / \mathrm{kwh}$. Each hour during the next ten months when the optimization is complete for the next 120 hour, the control signal is sent to control the energy and water according to the control rules described in section 2.3. 


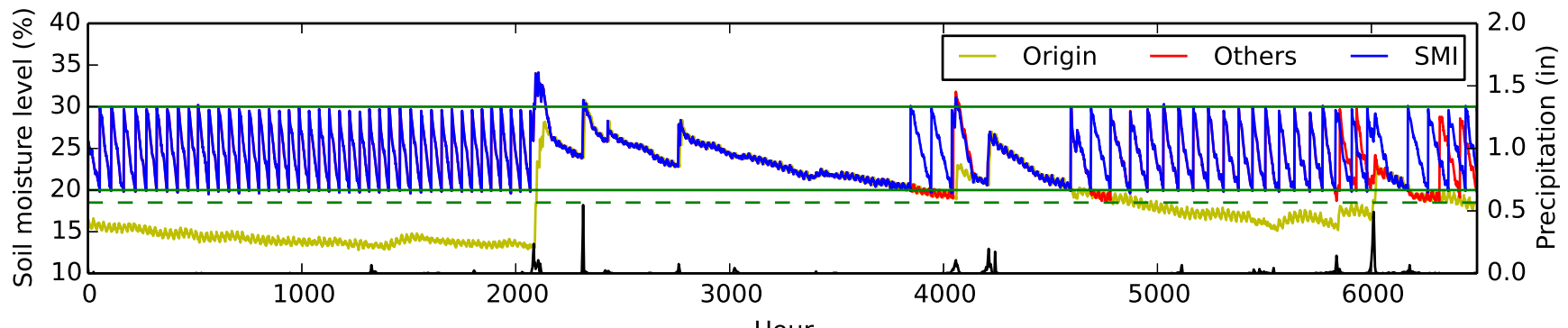

Figure 3: The irrigation performance. The ten month soil moisture traces for the origin, SMI and others (ODI, ODI-WOT \& Oracle) show that our proposed system saves water and energy for irrigation around $4000 \mathrm{~h}, 4700 \mathrm{~h}, 6000 \mathrm{~h}$ and $6300 \mathrm{~h}$. Especially, our system saves water and energy just hours before precipitation around $6000 \mathrm{~h}$.

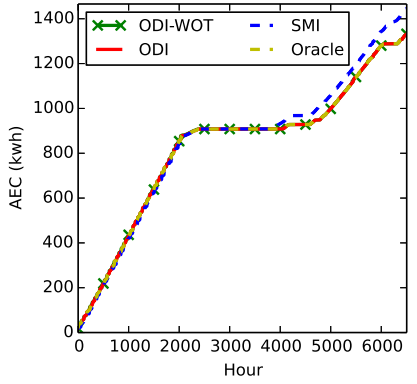

(a) Accumulated energy consumption.

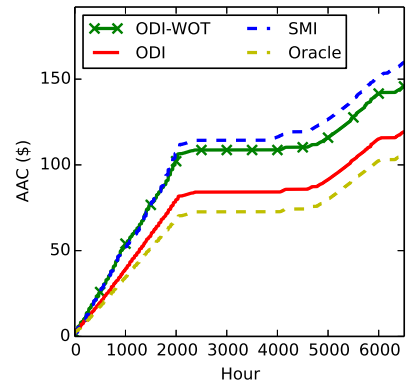

(b) Accumulated amortized cost.

Figure 4: Irrigation energy consumption and amortized cost.

\subsection{Baselines}

Three schedulers are implemented as baselines.

Soil Moisture based Irrigation (SMI). The scheduler [2] tries to irrigate the field once the soil moisture level at a certain layer drops below the irrigation start level until it reaches the target moisture level. Different from SMI, our design considers rain prediction in future to avoid over irrigation and reduce the total cost.

On-Demand Irrigation without TOU (ODI-WOT). The scheduling is the same to our design, ODI. However, it tries to irrigate the field with an averaged energy price from the public grid instead of using TOU model to reduce the energy cost

Oracle. The scheduler works the same as ODI, but it can always shift energy consumption to the minimal cost.

\subsection{Metrics}

Three metrics are used to evaluate the irrigation, including: 1), Accumulated Energy Consumption (AEC). The accumulated energy used to irrigate the field over an irrigation period. 2), Accumulated Amortized Cost $(A A C)$. The summation of solar energy and grid energy cost over an irrigation period. 3), Irrigation Performance (IP). The soil moisture level changes over time.

\subsection{Result}

Fig.4a shows the accumulated energy consumption. Our system starts saving energy and water from hour 3850. By the end of the evaluation, we save up to $7.97 \%$ of both resources compared to SMI.

Fig. $4 \mathrm{~b}$ shows the accumulated amortized cost for irrigation. Our proposed ODI is always better than SMI and very close to Oracle. When compared to SMI, ODI eventually saves $25.34 \%$ while Oracle $33.31 \%$. Even ODI-WOT is better than SMI. This is because when the demand comes at a specific time the TOU price is high from the grid. SMI utilize the energy at that time to irrigate while ODI-WOT shifts the energy caching to earlier low TOU times. Before hour
3850 , although all schedulers apply the same amount of energy (Fig.4a) and water (Fig. 3), our proposed method can utilize TOU model to minimize the amortized cost for farm owners (Fig. 4b).

\section{CONCLUSION}

In this paper, we proposed an on-demand irrigation scheduling system that minimizes the amortized cost to meet the water and energy demand. To estimate the energy demand, we propose an algorithm that considers future precipitation to avoid wasting energy and water just before precipitations. A novel penalty mechanism is designed to solve the solar energy over-prediction problem due to the inaccuracy of NWP. In the hourly scheduling for the next several days, the scheduler considers TOU pricing model to reduce water and energy use as well as the amortized cost.

\section{ACKNOWLEDGMENTS}

The authors would also like to thank the anonymous referees for their valuable comments and helpful suggestions. This project is supported by NSF grant CNS-1503590 and UMBC COEIT Strategic Plan Implementation Grant.

\section{REFERENCES}

[1] Peter Bauer, Alan Thorpe, and Gilbert Brunet. 2015. The quiet revolution of numerical weather prediction. Nature 525, 7567 (2015), 47-55.

[2] Garry L Grabow, Arjun Vasanth, Dan Bowman, Rodney L Huffman, and Grady L Miller. 2008. Evaluation of evapotranspiration-based and soil-moisture-based irrigation control in turf. In World Environmental and Water Resources Congress 2008: Ahupua'A. ASCE, 1-9.

[3] Zhichuan Huang, Ting Zhu, Yu Gu, David Irwin, Aditya Mishra, and Prashant Shenoy. 2014. Minimizing electricity costs by sharing energy in sustainable microgrids. In Proceedings of the 1st ACM Conference on Embedded Systems for Energy-Efficient Buildings. ACM, 120-129.

[4] Srinivasan Iyengar, Navin Sharma, David Irwin, Prashant Shenoy, and Krithi Ramamritham. 2014. SolarCast: a cloud-based black box solar predictor for smart homes. In Proceedings of the 1st ACM Conference on Embedded Systems for Energy-Efficient Buildings. ACM, 40-49.

[5] Aditya Mishra, David Irwin, Prashant Shenoy, Jim Kurose, and Ting Zhu. 2012. SmartCharge: cutting the electricity bill in smart homes with energy storage. In Proceedings of the 3rd International Conference on Future Energy Systems: Where Energy, Computing and Communication Meet. ACM, 29.

[6] Bernard Omodei. 2015. Accuracy and uniformity of a gravity-feed method of irrigation. Irrigation Science 33, 2 (2015), 121-130.

[7] Navin Sharma, Jeremy Gummeson, David Irwin, Ting Zhu, and Prashant Shenoy. 2014. Leveraging weather forecasts in renewable energy systems. Sustainable Computing: Informatics and Systems 4, 3 (2014), 160-171.

[8] Navin Sharma, Pranshu Sharma, David Irwin, and Prashant Shenoy. 2011. Predicting solar generation from weather forecasts using machine learning. In SmartGridComm, 2011 IEEE International Conference on. IEEE, 528-533.

[9] George Vellidis, Michael Tucker, Calvin Perry, Craig Kvien, and C Bednarz. 2008. A real-time wireless smart sensor array for scheduling irrigation. Computers and electronics in agriculture 61, 1 (2008), 44-50.

[10] Weigang Zhong, Zhichuan Huang, Ting Zhu, Yu Gu, Qingquan Zhang, Ping Yi, Dingde Jiang, and Sheng Xiao. 2014. ides: Incentive-driven distributed energy sharing in sustainable microgrids. In Green Computing Conference (IGCC), 2014 International. IEEE, 1-10. 